\title{
Study on interface characteristics and frost resistance of recycled concrete
}

\author{
Ying Liu ${ }^{1, *}$, Peng Peng ${ }^{1,2}$ \\ ${ }^{1}$ Research Institute of Highway, Ministry of Transport, Beijing 100088, China; \\ ${ }^{2}$ China Highway \& Transportation Society, Beijing 100029, China.
}

\begin{abstract}
The structural characteristics of recycled aggregate concrete and its damage mechanism have been analyzed in this paper. The recycled aggregate has been classified into three types through particle shaping and then frost resistance property of recycled concrete with different cement volume has been analyzed through the test. According to the test results, frost resistance property of the recycled concrete is $\mathrm{C}>\mathrm{B}>\mathrm{A}$, therefore, particle shaping can obviously improve quality and durability of the recycled coarse aggregate.
\end{abstract}

\section{Introduction}

With the rapid development of the society and the reconstruction of the infrastructure, the demand for concrete is increasing, and the treatment of a large number of demolished building wastes will bring some harm to the environment. According to the conservative estimation, annual average output for waste concrete in China is about $100,000,000$ tons. In addition to a small amount of concrete recycled to non-bearing structure such as subgrade base, the vast majority of concrete has been backfilled or piled up, which will pollute the environment and waste resources ${ }^{[1]}$. Besides, annual average production of concrete in China has been up to 1.5 to 2 billion cubic meters and quantity demanded for sand and gravel aggregate has exceeded 10 billion tons ${ }^{[2]}$. The exhaustive exploitation for sand and gravel has resulted in landslide and diversion of riverbed and damaged sustainable development of ecological environment in native area of the aggregate. Besides, the high-quality and natural aggregate (river sand and grait) has been exhausted in some regions. Looking for source of replaceable aggregate, disposing waste concrete and maintaining sustainable development of the environment have attracted more people to study recycling of the waste concrete.

Therefore, this paper put forward the basic idea of recycled aggregate concrete, and hopes to replace partially ordinary concrete with recycled aggregate concrete. It can not only meet the social needs, but also reduce the harm to the environment and realize the recycling of resources, and recycled aggregate concrete research also become the focus of social research nowadays. However, due to the large amount of old adhered mortar in recycled aggregate, it has certain defects in mechanical properties compared with ordinary concrete ${ }^{[3-5]}$.At present, the processing mechanism of recycled aggregate concrete technology is not perfect, and there are many bottlenecks that need to be broken in order to improve various mechanical performance indexes. All of these limit the development of recycled aggregate concrete to a large extent. So far, recycled aggregate concrete has been mostly used in some non-load-bearing components or road surfaces. The study on the damage mechanism and modification method of recycled aggregate concrete are of great significance for improving the destructive of recycled aggregate concrete and strengthen its application in engineering.

According to the related research, it is found that either the tensile failure or the compression failure of the recycled aggregate concrete is manifested by the fracture of the old adhered mortar and the interface transition zone. The initial micro cracks in the process are usually first appeared in the interface transition zone, and then extend to the mortar area. Therefore, the interface transition zone is the weakest link of recycled aggregate concrete, and the main factor causing this phenomenon is the existence of old mortar. To a large extent, the old mortar influences the difference in quantity, distribution and performance of interface transition zone. In recent years, many scholars have done research on recycled aggregate concrete, and have made a series of research achievements in improving the performance of recycled aggregate and the strength of recycled aggregate concrete. This paper will sort out and summarize these achievements in order to further expand and improve the regenerative aggregate concrete damage machine. It provides a reference for the research work of theory and modification.

\section{Mechanism of improving failure performance of recycled aggregate concrete}

The failure of recycled aggregate concrete block is mainly caused by tensile failure and compression failure. 


\subsection{Tensile failure}

At present, there are not many researches on the tensile failure of recycled aggregate concrete. In terms of its failure form, the failure surface of recycled aggregate concrete is mainly the fracture of mortar. Secondly, the interfacial transition zone between natural aggregate and new mortar or old mortar is damaged, while the fracture of recycled aggregate is relatively less. Existing studies show that the tensile strength of recycled aggregate concrete is related to many factors, such as replacement rate, mix ratio and water-cement ratio. The split tensile test of Tabsh et al. ${ }^{[6]}$ shows that the splitting tensile strength of recycled aggregate concrete is related to the mixing ratio of recycled aggregate concrete, which is generally $10 \% \sim 25 \%$ lower than that of ordinary concrete. Ikeda et al. ${ }^{[7-8]}$ found that the tensile strength of recycled aggregate concrete was $6 \% \sim 10 \%$ lower than that of ordinary concrete, Sageo-Crent-sil et al. ${ }^{[9]}$ test show that The ratio between tensile strength and compressive strength of recycled aggregate concrete is slightly higher than that of ordinary concrete, Gupta ${ }^{[10]}$ test find when water-cement ratio is low, the tensile strength of recycled aggregate concrete is lower than normal concrete, and water cement ratio is high, the tensile strength of recycled aggregate concrete is higher than that of ordinary concrete.

\subsection{Compression failure}

As compression is the main performance of concrete, it is common to study the compressive failure and compressive strength of recycled aggregate concrete. The researches of Jianzhuang Xiao et al, ${ }^{[11]}$ show that the compressive strength of recycled aggregate concrete is closely related to the replacement rate, water-cement ratio and age, etc., and the failure surface of recycled aggregate concrete basically starts from the bond failure between coarse aggregate and hydrogels. Xu-ping $\mathrm{Li}^{[12]}$ research on mechanical performance of recycled aggregate concrete suggests that modulus of elasticity of recycled aggregate concrete is lower than the ordinary concrete and peak strain and brittleness are bigger than the ordinary concrete, and the failure form of prism is longitudinal splitting under uniaxial compression. Xuebing Zhang et al. ${ }^{[13]}$ prove that water-cement ratio have a great influence on compressive strength of recycled aggregate concrete with different mixing ratios. Throughout the existing studies, the compressive failure of recycled aggregate concrete test blocks is mainly caused by oblique cracks, and a few of them are caused by local crushing. Most of the fracture surfaces of recycled aggregate concrete are old mortar fracture and interface transition zone failure, namely interface transition zone between natural aggregate and new mortar or old mortar.

\section{Structural characteristics of recycled concrete interface}

Due to limited manufacturing technology and cost, the recycled aggregate is generally complex containing multiple structural defects. The old interface between original aggregate and hardened cement paste is the largest defect inside the complex and has important influences on mechanical property and durability of the recycled concrete. Actually, concrete is a composite material which is formed by the aggregate (coarse aggregate + fine aggregate) uniformly distributed in cement paste, the aggregate is non-continuous reinforced phase and cement paste is continuous basis material phase (medium) ${ }^{[14-15]}$. The concrete interface includes coarse aggregate-cement mortar interface and fine aggregate-cement mortar interface. The interface in concrete is a loosened and porous structure, in which massive enrichment and orientation of $\mathrm{Ca}(\mathrm{OH})_{2}$ exists. Therefore, it is the weakest part in concrete and has obvious influences on mechanical property and durability ${ }^{[16]}$. Due to large surface area of the coarse aggregate, the interface between coarse aggregate and cement paste will generate large-scale structural defect, therefore, its damage effect on concrete property is greatly larger than structural component of interface between fine aggregate and cement paste. On this basis, influences of coarse aggregate-cement paste interface on concrete property are generally studied only. That is to say, the concrete is deemed as the composite material that is formed in cement mortar by uniformly distributed coarse aggregate ${ }^{[17-18]}$. The coarse aggregate is non-continuous reinforced phase and cement paste is continuous basis material phase (medium).

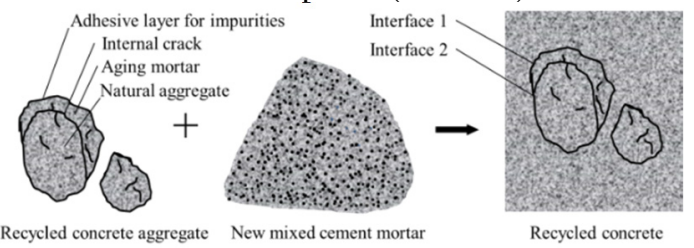

Figure 1 Diagram of recycled concrete aggregate parceled by cement mortar

There are two important interfaces in recycled concrete: first, interface between natural aggregate in recycled aggregate and old cement mortar, as shown in Figure 1; second, interface between recycled aggregate and new mixed cement mortar, as shown in Figure 1. During cement hydration and hardening process, there is not any chemical reaction between cement and aggregate, but combination between cement mortar and aggregate is physical. Besides, according to micro-examination, during concrete mixing, water film forms around coarse aggregate and high water cement ratio results in relatively large crystallized product around coarse aggregate interface, thus to form a more porous structure than ordinary cement base phase or mortar. Therefore, the interface between aggregate and cement mortar in concrete is relatively weak and will easily generate small crack and start destructive development from interface joint part under stress. During crushing process for waste concrete, the crash and damage will result in small cracks inside recycled aggregate, while the relatively weak interface between aggregate and mortar tends to fall off easily. However, for interface 2, due to coarse surface, the recycled aggregate will be covered by waste dust and impurities, which has seriously influence cementation between cement mortar and recycled aggregate and finally resulted in loose interface. 


\section{Analysis of frost resistance test for recycled coarse aggregate concrete}

\subsection{Test principles and process}

The rapid freezing-thawing method has been applied in this test to measure mass loss rate and relatively dynamic modulus of elasticity of the sample. Test process:

(1) Sample size: $100 \mathrm{~mm} \times 100 \mathrm{~mm} \times 400 \mathrm{~mm}$; avoid hydrophobic release agent, maintain to valid period of standard and implement water curing 4 days in advance;

(2) Wipe the surface and dry, and record initial weight and modulus of elasticity;

(3) Put the sample into sample box, ensure liquid level $5 \mathrm{~mm}$ higher than the sample, and then conduct freezing and thawing test.

\subsection{Classification for recycled aggregate}

Type II recycled coarse aggregate, indicated by A; Quasi type I recycled coarse aggregate, indicated by B; Type I recycled coarse aggregate, indicated by $\mathrm{C}$, and ordinary concrete, indicated by NA. In the test, replacement rate for recycled coarse aggregate is $50 \%$.

\subsection{Test results}

Please refer to Standard for Test Methods of Long-term Performance and Durability of Concrete to measure mass loss rate and relatively dynamic modulus of elasticity of the sample. See Figure 2 for results. Where, the cement content is taken as $350 \mathrm{~kg} / \mathrm{m}^{3}, 400 \mathrm{~kg} / \mathrm{m}^{3}$ and $500 \mathrm{~kg} / \mathrm{m}^{3}$ respectively.

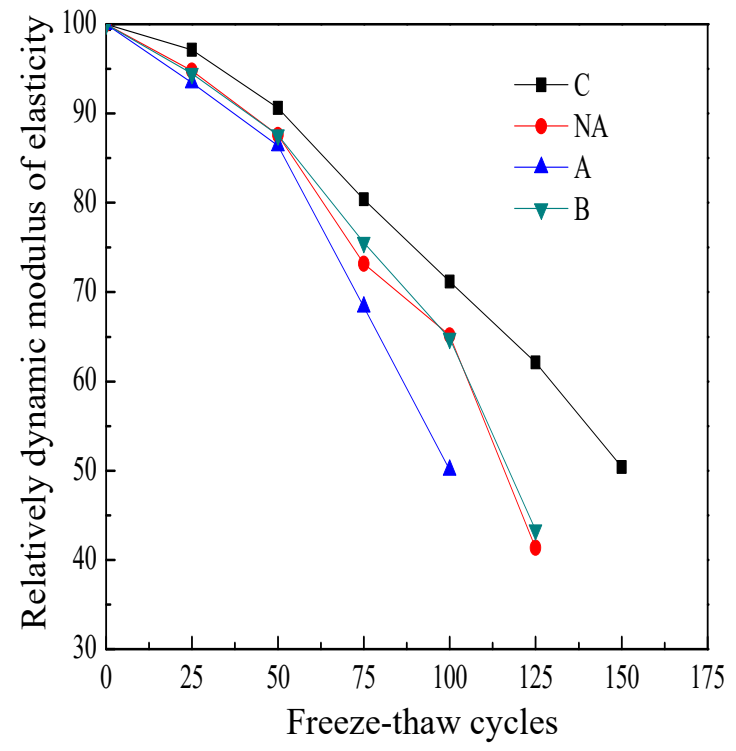

(a) Cement content $350 \mathrm{~kg} / \mathrm{m}^{3}$

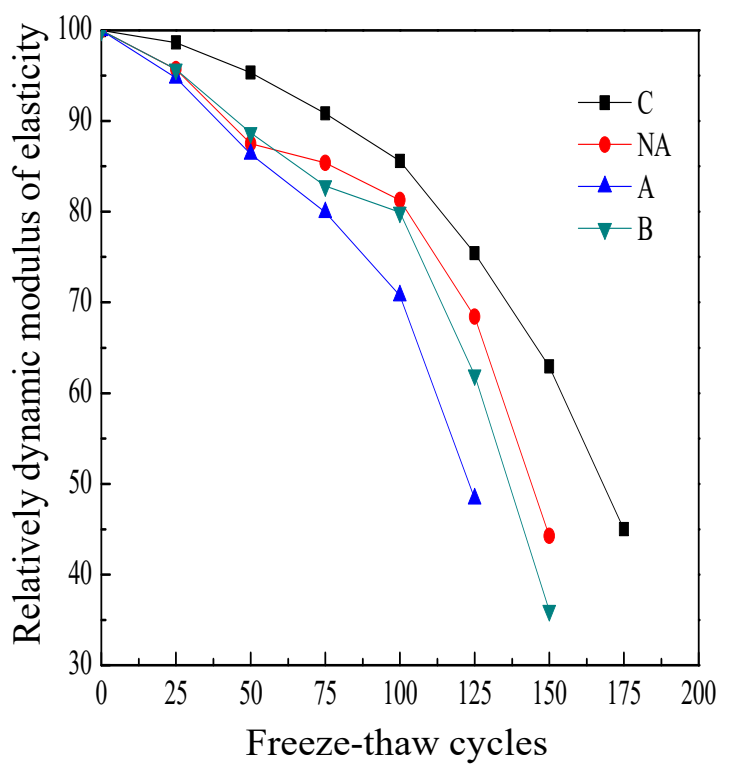

(b) Cement content $400 \mathrm{~kg} / \mathrm{m}^{3}$

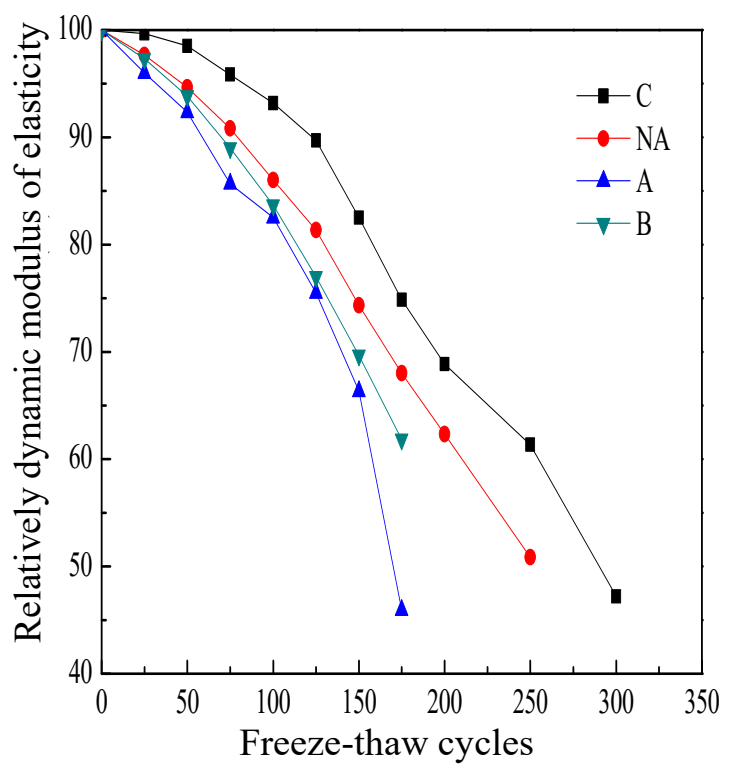

(c) Cement content $500 \mathrm{~kg} / \mathrm{m}^{3}$

Figure 2 Diagram of test data about relatively dynamic modulus of elasticity for recycled concrete with different concrete contents

\subsection{Results analysis}

The influence and function mechanism of particle shaping for recycled coarse aggregate have been analyzed and test conclusions have been drawn as below:

(1) The frost resistance property is the worst and worse than ordinary concrete for A recycled concrete, approximate to ordinary concrete for $\mathrm{B}$ recycled concrete, the best and better than ordinary concrete for $\mathrm{C}$ recycled concrete. Compared with A recycled concrete, freezing and thawing cycles of $\mathrm{B}$ and $\mathrm{C}$ recycled concrete have increased 25 and 25 to 125 times respectively.

(2) As replacement rate of recycled coarse aggregate increases, frost resistance property tends to decline for A and $\mathrm{B}$ recycled concrete, but increase and then decline for 
C recycled concrete.

\section{Conclusions}

(1) Although reinforcement technology for recycled aggregate has been largely developed and adherent mortar on surface of recycled aggregate has been removed based on above technologies to a large extent, the adherent mortar has not been eliminated completely and the residual adherent mortar on surface of recycled coarse aggregate will increase multiple interfaces in recycled concrete, which will seriously influence mechanical property and durability of the recycled concrete. The recycled concrete is a multi-phase complex in which random distribution of multiple interfaces has increased difficulty in studying interface structure and hindered study on damage mechanism for property of recycled concrete.

(2) The frost resistance property of the recycled coarse aggregate concrete is $\mathrm{C}>\mathrm{B}>\mathrm{A}$. Therefore, particle shaping can obviously improve the quality of the recycled coarse aggregate and improve the durability of recycled concrete.

\section{References}

1. XIAO jian-zhuang, LI jia-bin, SUN Zhen-ping, et al. Study on Compressive Strength of Recycled Aggregate Concrete [J].Journal of Tongji University: Natural Science, 2004,32(12):1558-1561

2. ZHANG Xue-bing, DENG Shou-chang, DENG $\mathrm{Xu}$-hua, et al. Experimental Research on a Few Main Factors Influencing Strength of the Recycled Concrete [J].Natural Science Journal of Xiangtan University,2005,27(1)129-133.

3. OTSUKI N,MIYAZATO S,YODSUDJAI W. Influence of Recycled Aggregate on Interfacial Transition Zone, Strength, Chloride Penetration and Carbonation of Concrete[J]. Journal of Materials in Civil Engineering, 2003, 15(5):443-451.

4. TOPCU I B, SENGEL S. Properties of Concretes Produced with Waste Concrete Aggregate [J]. Cement and Concrete Research, 2004, 34(8); 1307-1312.

5. LI Juan. Influence of Mortar Adhesive to Recycled Aggregate on Strength of Concrete and Reserch of Lime-fly Ash Stablizied Recycle Aggregate [D]. Nanjing; Hohai University,2005.

6. TABSH S W, ABDELFATAH AS. Influence of Recycled Concrete Aggregates on Strength Properties of Concrete [J].Construction and Building Materials, 2009, 23(2):1163-1167

7. IKEDA T, YAMANE S. Strengths of Concrete Containing Recycled Aggregate[C]//KASAI Y. Proceedings of the Second International RILEM Symposium on Demolition and Reuse of Concrete and Masonry-Tokyo: CRC Press, 1988:585-594

8. RAVINDRARAJAH R S, TAM C T. Recycled Concrete as Fine and Coarse Aggregates in
Concrete [J]. Magazine of Concrete Research, 1987, 39(141):214-220

9. SAGOE-CRENTSIL K, BROWN T, TAYLOR AH. Performance of Concrete Made with Commercially Produced Coarse Recycled Concrete Aggregate [J].Cement and Concrete Research, 2001.31(5):707-712

10. GUPTA S M. Strength Characteristics of Concrete Made with Demolition Waste as Coarse Aggregate[C]// Phoenix Pub House. Proceedings of the International Conference on Recent Development in Structure Engineering, New Delhi: Phoenix Pub House, 2001:364-373.

11. XIAO jian-zhuang, LI jia-bin, SUN Zhen-ping, et al. Study on Compressive Strength of Recycled Aggregate Concrete [J].Journal of Tongji University: Natural Science, 2004, 32(12):1558-1561.

12. LI Xu-ping. Study on Mechanical Properties of Recycled Aggregate Concrete (I)-Behaviour under Uniaxial Compression [J].Journal of Building Materials, 2007, 10(5):598-603.

13. ZHANG Xue-bing, DENG Shou-chang, DENG Xu-hua, et al. Experimental Research on a Few Main Factors Influencing Strength of the Recycled Concrete $[\mathrm{J}]$. Natural Science Journal of Xiangtan University, 2005, 27(1)129-133.

14. LI Qiu-yi, LI Yun-xia, ZHU Chong-ji . The influence of a particle shape correcting technique in properties of recycled coarse aggregate [J] . Materials Science \& Technology, 2005,13(6):579-281,585.

15. ZHU Chong-ji, LI Qiu-yi, LI Yun-xia. The Affection by Aggregate-shaping on Durability of the Recycled Aggregate Concrete[J].China Building Materials Science \& Technology,2007,16(3):6-10.

16. CUI Zheng-long, TONG Hua-bin, WU Xiang-yu, et al. Research on Autoclave Curing Effect of the Mechanical Properties of Recycled Aggregate Concrete[J].Bulletin of the Chinese Ceramic Society ,2014,33(3):596-599.

17. ZHAO Wu, FENG Zhou-xu. Research on the Strengthening Mechanism of Vibration Mixing of Recycled Concrete [J].Concrete, 2006(8):17-20.

18. ABBASA, FATHIFAZLG, POURNIERB, et al. Quantification of the Residual Mortar Content in Recycled Concrete Aggregates by Image Analysis[J].Materials Characterization,2009,60(7):716-728. 